\title{
BP Neural Network Algorithms for Fault Diagnosis of Microwave Components
}

\author{
Gao Kun ${ }^{1}$, Wang Aimin ${ }^{1}$ and Ge Yan ${ }^{1}$ \\ ${ }^{1}$ Department of Mechanical Engineering, Beijing Institute of Technology, Beijing, China
}

\begin{abstract}
Intelligent diagnosis is the main trend of modern fault diagnosis technology. The emergence of artificial neural network technology provides a new way for this kind of intellectualization. Aiming at the problem of microwave module fault diagnosis, an intelligent fault diagnosis method based on BP(Back Propagation) neural network is proposed in this paper. In this paper, the process of determining the neural network model and the operation flow of BP algorithm are introduced, and the network is trained with training samples. By applying the neural network model to an AQ module for testing, the feasibility, accuracy and efficiency of the fault diagnosis of the microwave module are verified, which provides a new method for intelligent fault diagnosis of this kind of microwave module.
\end{abstract}

\section{Introduction}

In recent years, fault diagnosis technology has developed at an unprecedented high speed and attracted many researchers. Fault diagnosis technology is a kind of technology that detects the abnormal situation by monitoring the state parameters of equipment or products, and then analyses and diagnoses the causes of the abnormal situation. Its purpose is to find the potential failure of products by virtue of all current innovative technologies, so as to prevent the product quality accidents. At present, fault diagnosis, as an independent and interdisciplinary integrated information processing technology, has developed into a hot research direction in the field of control.

Artificial Neural Network is a widely used model. Its theoretical development has been very mature, and many breakthroughs have been made, which has attracted great attention from the academic circles at home and abroad. As a new modeling technology, BP neural network has been used by technicians from all walks of life. In the aspect of fault diagnosis, the network model is also widely used.

Fault diagnosis has been widely applied in the field of engineering equipment repair and maintenance. Dai Min et al. proposed a fault diagnosis method based on fuzzy weighted colored network and BP neural network, aiming at solving the problems that the parameters of traditional knowledge reasoning fault diagnosis methods often depend on experts' experience and so the inaccuracy and learning obstacles exist. Li Ning et al. studied the fault reasoning model of aircraft switched reluctance power generation system. By introducing memory module, the algebraic loop problem in the past simulation process was solved. Then the output voltage signal of generator was decomposed by EMD algorithm. The standard deviation of the decomposed frequency signals of each layer is taken as the characteristic quantity, and then the neural network is trained, and the fault reasoning model of switched reluctance power generation system is obtained. M. Demtgul et al. studied and designed a pneumatic expert system for fault diagnosis. S.C.Liu and S.Y.Liu developed and designed a fault diagnosis expert system based on heuristic rules for hydropower units. R.K. Autar developed a diesel engine fault diagnosis system. J. David et al. designed and developed an expert system for identifying and diagnosing the condition of steam turbines, which can monitor the vibration faults better.

In the production process of microwave products, the process of measurement is the most important step, which directly affects the quality of microwave products and the completion of tasks. However, in the traditional measurement process, the fault diagnosis problems of the microwave components under test are mostly judged by the inspectors according to the test results. The diagnostic efficiency is low and the flow of the tested parts is lagging behind. In order to avoid this situation, this paper proposes an intelligent fault diagnosis method based on BP neural network for microwave components of Automatic Test Unit, aiming at improving the efficiency of fault diagnosis and realizing the high efficiency and intelligence of fault location.

\section{Neural Network Model Based on BP Algorithms}


Neural network is an operation model, which consists of a large number of nodes (or neurons) connected with each other. Each node represents a specific output function, called an Activation Function. The connection between two nodes represents a weighted value of the signal passing through the connection, which is called the weight.And it is equivalent to the memory of the artificial neural network.

To solve the problem of microwave module fault diagnosis, a neural network model based on BP algorithm is proposed in this paper. Figure 1 shows a schematic diagram of the neural network model.

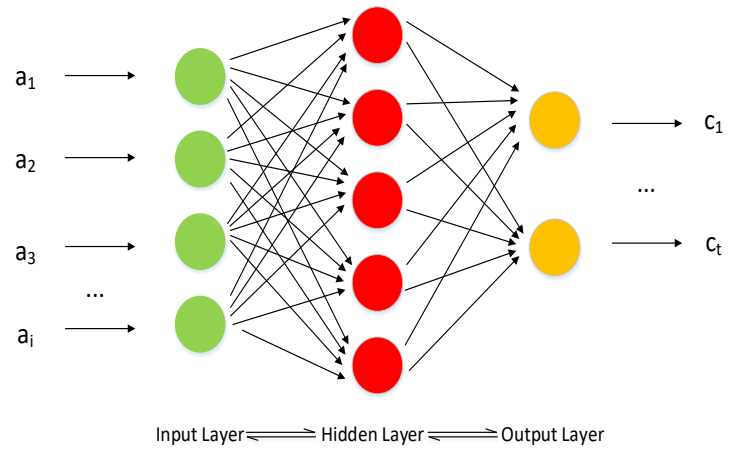

Figure 1. Neural Network Model Structure Based on BP Algorithms.

As shown in Fig. 1, the network model consists of three parts: input layer, hidden layer and output layer. The input layer is used to input the information of fault phenomena, and convert the fault phenomena into the mode of multi-dimensional vectors. The dimensions of vectors depend on the number of fault phenomena identified and extracted. Increasing the hidden layer can reduce the network error and improve the accuracy, but it will also complicate the network, thus increasing the training time of the network and leading to the phenomenon of over-fitting. Therefore, when designing the network model, priority should be given to three layers of network, i.e., only one hidden layer. Then, by adjusting the number of nodes in the hidden layer, error can be lowered. The input layer is used to output fault reason information for diagnosis. The dimension of the output vector depends on the number of fault reasons identified and extracted. The diagnosis result can be obtained by converting the output multidimensional vector into fault reason information.

The process of network model learning and training includes forward propagation and backward feedback of errors within the network. Its purpose is to minimize network errors by adjusting the weights and thresholds of connections within the network. The specific operation flow is as follows:

1) Forward propagation. After initializing the network model, the training set of known diagnostic results is input into the model one by one for forward propagation operation. The specific calculation method is as follows:

output of hidden layer:

$$
b_{j}=f\left(\sum_{\mathrm{i}=1}^{\mathrm{N}} \omega_{i j} a_{i}+\theta_{j}\right)
$$

output of output layer:

$$
c_{t}=f\left(\sum_{j=1}^{N} v_{j t} b_{j}+r_{t}\right)
$$

Formula: ${ }^{b_{j}}$ is the actual output of the $\mathrm{j}$-th neuron in the hidden layer. ${ }^{c_{t}}$ is the actual output of the t-th neuron in the output layer. $\theta_{j}$ is the threshold from input layer to hidden layer. $r_{t}$ is the threshold from hidden layer to output layer. $\omega_{i j}$ is the connection weight from input layer to hidden layer. $v_{j t}$ is the connection weight from the hidden layer to the output layer.

When the output layer calculates the actual output, the calculation error is obtained by comparing with the expected output in the known results. The specific calculation formula is as follows:

$$
E=\frac{1}{2} \sum_{i=1}^{N}\left(d_{t}-c_{t}\right)
$$

Formula: $N$ is the number of neurons in the front layer of the neuron. $d_{t}$ is the expected output. $E$ isthe error. Here make $\boldsymbol{d}_{t}-\boldsymbol{c}_{t}=\boldsymbol{e}_{t}$, so $E$ can be expressed as:

$$
E=\frac{1}{2} \sum_{i=1}^{N} e_{t}^{2}
$$

2) Backward feedback. According to the error of forward propagation calculation, the weight value $\omega_{i j}$ between input layer and hidden layer and the weight value $v_{j t}$ between hidden layer and output layer are adjusted. The updated formula is:

$$
\left\{\begin{array}{c}
\omega_{i j}=\omega_{i j}+\eta b_{j}\left(1-b_{j}\right) a_{i} \sum_{\mathrm{i}=1}^{\mathrm{N}} v_{j t} e_{t} \\
v_{j t}=v_{j t}+\eta b_{j} e_{t}
\end{array}\right.
$$

Adjust threshold $\theta_{j}$ between input layer and hidden layer and threshold $r_{t}$ between hidden layer and output layer according to error. The updated formula is:

$$
\left\{\begin{array}{c}
\theta_{j}=\theta_{j}+\eta b_{j}\left(1-b_{j}\right) \sum_{i=1}^{\mathrm{N}} v_{j t} e_{t} \\
r_{t}=r_{t}+\eta e_{t}
\end{array}\right.
$$

This is the process of error back propagation. The goal is to minimize the error function, i.e. $\min E$. The above two formulas are calculated by gradient descent method.

\section{Solution}


Microwave module is one of the key components in active phased array radar. There are many kinds of components and complex fault conditions. The combination of multiple fault phenomena will form a nonlinear coupling relationship with multiple fault causes, which increases the difficulty of locating the fault causes according to the fault phenomena. In this section, the BP neural network model is used to realize the fault diagnosis of microwave components. The training flow of the algorithm is shown in Fig. 2.
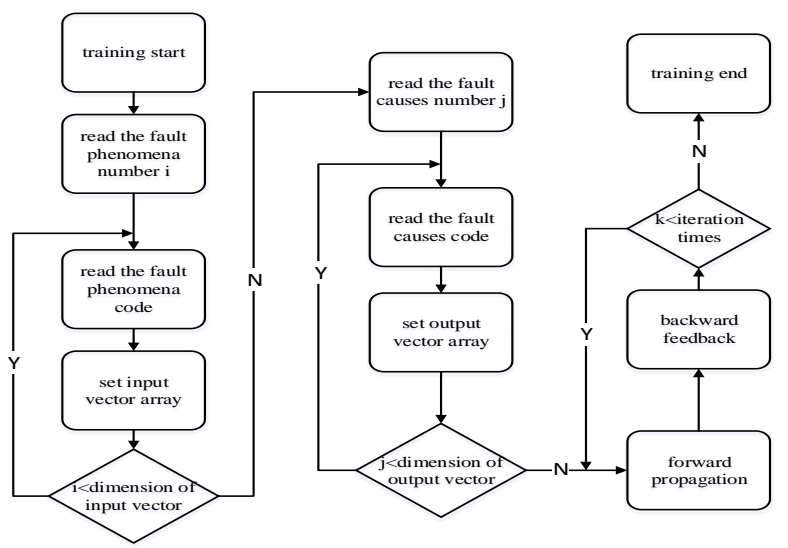

Figure 2. BP Neural Network Model Training Process.

As can be seen from Fig. 2, there are four main steps in the process of neural network training for fault diagnosis of microwave components.

Step 1: Set up an array of input vectors to convert the fault phenomenon information in the sample file into multi-dimensional input vectors.

Step 2: Set up an array of output vectors to convert the fault causes information in the sample file into multidimensional output vectors.

Step 3: Forward propagation, the input vectors are input into the network model for layer-by-layer operation, and the actual output vectors are compared with the expected output vectors to obtain the calculation error.

Step 4: Backward feedback, according to the calculation error obtained in the previous step, adjusts the connection weight and threshold between the nodes of each unit in the network.

\subsection{Determine input and output vectors.}

According to the analysis of fault mechanism and the knowledge of experts in the field, we can get the common fault phenomena and main fault causes in the testing process of microwave components, which are shown in Tables 1 and 2, respectively.

Table 1. Common Faults Phenomenon of Microwave Modules

\begin{tabular}{|c|c|c|}
\hline Serial number & Code & Fault phenomenon \\
\hline 1 & $\mathrm{Y}_{1}$ & Substandard power \\
\hline 2 & $\mathrm{Y}_{2}$ & Excessive power \\
\hline 3 & $\mathrm{Y}_{3}$ & Emission phase overshoot \\
\hline 4 & $\mathrm{Y}_{4}$ & Emission phase discretization \\
\hline 5 & $\mathrm{Y}_{5}$ & Substandard gain \\
\hline
\end{tabular}

\begin{tabular}{|c|c|c|}
\hline 6 & $\mathrm{Y}_{6}$ & Excessive gain \\
\hline 7 & $\mathrm{Y}_{7}$ & $\begin{array}{c}\text { Over-tolerance of receiving gain } \\
\text { fluctuation }\end{array}$ \\
\hline 8 & $\mathrm{Y}_{8}$ & Receiver phase overshoot \\
\hline 9 & $\mathrm{Y}_{9}$ & All channel no transceiver \\
\hline 10 & $\mathrm{Y}_{10}$ & Single channel no transceiver \\
\hline 11 & $\mathrm{Y}_{11}$ & Wrong version number \\
\hline 12 & $\mathrm{Y}_{12}$ & Wrong temperature return value \\
\hline
\end{tabular}

Table 2. Common FaultsCauses of Microwave Modules

\begin{tabular}{|c|c|c|}
\hline Serial number & Code & Fault Causes \\
\hline 1 & $\mathrm{R}_{1}$ & Fault of power amplifier module \\
\hline 2 & $\mathrm{R}_{2}$ & Fault of L board \\
\hline 3 & $\mathrm{R}_{3}$ & Digital channel failure \\
\hline 4 & $\mathrm{R}_{4}$ & Circulator short circuit \\
\hline 5 & $\mathrm{R}_{5}$ & Failure of integrated panel \\
\hline 6 & $\mathrm{R}_{6}$ & Omega fault of power amplifier \\
\hline 7 & $\mathrm{R}_{7}$ & Power leakage welding \\
\hline 8 & $\mathrm{R}_{8}$ & Low noise amplifier \\
\hline 9 & $\mathrm{R}_{9}$ & RF cable failure \\
\hline 10 & $\mathrm{R}_{10}$ & 298 cable fault \\
\hline 11 & $\mathrm{R}_{11}$ & Local oscillator cable fault \\
\hline
\end{tabular}

After extracting the fault feature, the number of nodes in the input and output layers of the neural network model is determined, which is consistent with the number of fault phenomena and the types of fault causes, respectively. At the same time, according to the empirical formulas and experiments, it is shown that selecting 10 nodes of hidden layer unit can not only ensure the accuracy of fault diagnosis of network model, but also avoid too long training time and over-fitting phenomenon. Sigmoid function is chosen as the excitation function, and its formula is as follows:

$$
f(x)=\frac{1}{1+e^{-x}}
$$

It is necessary to convert the detected raw data into a multi-dimensional vector pattern that can be recognized by the network model. By comparing the current value of the state to be inspected with the presupposed normal value, the ratio is used as the basis for judging whether the fault phenomenon occurs or not. The judgment formula is defined as:

$$
F\left(x_{i}\right)=s_{T}\left(x_{i}\right) / s_{R}\left(x_{i}\right)
$$

Formula: $S_{T}\left(X_{i}\right)$ is the value of the pending state parameter relative to $x_{i} . S_{R}\left(x_{i}\right)$ is the normal state parameter value relative to $x_{i}$. The ratio $F\left(x_{i}\right)$ of the two values is compared with the threshold value. If it exceeds the threshold value, the corresponding location 
of the fault feature is input as 1 , that is, the fault phenomenon occurs, otherwise it is 0 , that is, the fault phenomenon does not occur. After the input and output vectors are determined, the fault phenomena information in the training samples is converted into input vectors in the above way, and the fault causes information is converted into output vectors, and then a vector group similar to that shown in Table 3 is obtained. The graph shows that the corresponding fault phenomena of Y1in the input vectors occur and the corresponding faults cause is $R_{2}$.

Table 3. Input and output vectors

\begin{tabular}{|c|c|c|c|c|c|c|c|c|c|}
\hline \multicolumn{5}{|c|}{ input vector } & \multicolumn{5}{c|}{ output vector } \\
\hline$Y_{1}$ & $Y_{2}$ & $\cdots$ & $Y_{11}$ & $Y_{12}$ & $R_{1}$ & $R_{2}$ & $\cdots$ & $R_{10}$ & $R_{11}$ \\
\hline 1 & 0 & $\cdots$ & 0 & 0 & 0 & 1 & $\cdots$ & 0 & 0 \\
\hline
\end{tabular}

\subsection{Forward propagation}

Through the above way, the fault information in training samples is converted into input and output vectors which can be recognized by network model. The input vectors are calculated according to the formula layer by layer. After the actual output vectors are obtained, the calculation errors of the model are compared with the expected output vectors.

\subsection{Backward feedback}

After calculating the error of the network model, update the connection weights and thresholds value between the output layer and the hidden layer, the hidden layer and the input layer according to the formula. When the number of iterations reaches the upper limit, the training of the input and output vectors for the network model is stopped, and the training of the neural network model is completed after all the training samples have completed the iteration.

\subsection{Fault diagnosis}

When the neural network is trained by BP algorithm, it can be used for intelligent fault diagnosis of microwave components. The process of intelligent fault diagnosis of microwave components involved in this paper is shown in Fig. 3:

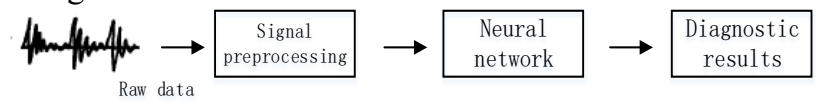

Figure 3. Fault diagnosis process

Step 1: Obtain the original data in the microwave module testing process, and convert it into multidimensional input vectors which can be recognized by the neural network model.

Step 2: Input vector is input into the trained neural network model for operation.

Step 3: Multidimensional output vectors are obtained by neural network operation. The results of output vectors determine what fault causes the fault phenomena.

\section{Example Verification}

According to the above BP neural network algorithm for fault diagnosis of microwave components, a simulation example has been performed in VS20100. Firstly, according to the above discussion, a three-layer neural network model is established. The number of nodes in the input layer is 12 , the number of nodes in the hidden layer is 10 , and the number of nodes in the output layer is 11 . The Sigmoid function is chosen as the excitation function. After establishing the network model, input the training sample set as shown in Table 4 below.

Table 4. Training Sample Data Set

\begin{tabular}{|c|c|c|c|c|c|c|c|c|}
\hline \multirow{2}{*}{$\begin{array}{c}\text { Sample } \\
\text { serial } \\
\text { number }\end{array}$} & \multicolumn{4}{|c|}{ Input vector } & \multicolumn{4}{|c|}{ Output vector } \\
\hline & $\mathrm{Y}_{1}$ & $\mathrm{Y}_{2}$ & $\cdots$ & $\mathrm{Y}_{12}$ & $\mathrm{R}_{1}$ & $\mathrm{R}_{2}$ & $\cdots$ & $\mathrm{R}_{11}$ \\
\hline 1 & 1 & 0 & $\cdots$ & 0 & 1 & 0 & $\cdots$ & 0 \\
\hline 2 & 0 & 1 & $\cdots$ & 0 & 0 & 1 & $\cdots$ & 0 \\
\hline$\vdots$ & $\vdots$ & $\vdots$ & $\vdots$ & $\vdots$ & $\bar{x}^{\prime}$ & $\vdots$ & $\vdots$ & $\frac{1}{\vdots}$ \\
\hline 11 & 0 & 0 & $\cdots$ & 0 & 0 & 0 & $\cdots$ & 0 \\
\hline 12 & 0 & 0 & $\cdots$ & 1 & 0 & 0 & $\cdots$ & 1 \\
\hline
\end{tabular}

After finite iterations, the neural network model learns the relationship between fault phenomena and fault causes in training samples, and achieves the fitting of the non-linear relationship between them. Based on the trained network model, the intelligent fault diagnosis test is carried out, and the results shown in Table 5 are as follows:

Table 5. Fault Diagnosis Test Results

\begin{tabular}{|c|c|c|c|}
\hline $\begin{array}{c}\text { Fault } \\
\text { phenomenon }\end{array}$ & $\begin{array}{c}\text { Expected } \\
\text { output }\end{array}$ & $\begin{array}{c}\text { Actual } \\
\text { output }\end{array}$ & $\begin{array}{c}\text { Diagnostic } \\
\text { results }\end{array}$ \\
\hline $\begin{array}{c}\text { Substandard } \\
\text { power }\end{array}$ & 1 & 0.98364 & $\begin{array}{c}\text { Fault of power } \\
\text { amplifier } \\
\text { module }\end{array}$ \\
\hline $\begin{array}{c}\text { Emission phase } \\
\text { overshoot }\end{array}$ & 1 & 0.93247 & $\begin{array}{c}\text { Circulator } \\
\text { short circuit }\end{array}$ \\
\hline $\begin{array}{c}\text { Wrong version } \\
\text { number }\end{array}$ & 1 & 0.948234 & $\begin{array}{c}\text { Digital channel } \\
\text { failure }\end{array}$ \\
\hline $\begin{array}{c}\text { Substandard } \\
\text { gain }\end{array}$ & 1 & 0.973542 & $\begin{array}{c}\text { Failure of } \\
\text { integrated } \\
\text { panel }\end{array}$ \\
\hline $\begin{array}{c}\text { All channel no } \\
\text { transceiver }\end{array}$ & 1 & 0.954561 & $\begin{array}{c}\text { RF cable } \\
\text { failure }\end{array}$ \\
\hline $\begin{array}{c}\text { Receiver phase } \\
\text { overshoot }\end{array}$ & 1 & 0.994672 & $\begin{array}{c}\text { Fault of L } \\
\text { board }\end{array}$ \\
\hline $\begin{array}{c}\text { Emission phase } \\
\text { discretization }\end{array}$ & 1 & failure \\
\hline
\end{tabular}

Table 5 shows that the trained neural network model can be used to diagnose the faults of microwave components, and the average accuracy of the diagnostic results is more than $95 \%$.

\section{Summary}

(1) An intelligent fault diagnosis method based on BP neural network is proposed for microwave module fault diagnosis.

(2) By storing historical fault case information in each neuron of the network, the network structure can be dynamically adjusted, which makes the whole model 
have good robustness and fault tolerance, and improves the adaptability and stability of the model to a certain extent.

(3) The coupling relationship between fault phenomenon and fault cause in microwave module fault diagnosis is approached through BP neural network's highly fit non-linearity ability, so as to locate new faults quickly to their causes.

\section{References}

1. Lei Jiyao et al. Engineering signal processing technology. Chongqing: Chongqing University Press, 1990(in Chinese)

2. Liu Ling. Zhangxi. Wang Linna. Current Situation and Development of Fault Diagnosis Technology [Journal Papers] - Electronic Testing 2016 (2) (in Chinese)

3. Zhang Xinhai.Lei Yong. Application of BP Neural Network in Mechanical Fault Diagnosis. Northwest Polytechnic University: School of Power and Energy, 2008(in Chinese)

4. Dai Min. Xie Chun. Fault Diagnosis of Aircraft Engine Based on Fuzzy Weighted Nonferrous
Network and BP Neural Network [Journal Papers] Science, Technology and Engineering. 2012(in Chinese)

5. Li Ning, Lei Hongli, Han Jianding, Zhu Xihua. Research on Fault Reasoning Model of Aircraft Switched Reluctance Generation System [Journal Paper] - Power Supply Technology. 2011 (5) (in Chinese)

6. M.Demtgul, I.N.Tansel and S.Taskin.Fault diagnosis of pneumatic systems with artificial neural network Algorithms, Expert Systems with Applications,2009,36(7),10514-10519.

7. S.C.Liu,S.Y.Liu,in:An Efficient Expert System for Machine Fault Diagnosis Source.International Journal of Advanced Manufacturing Technology.2003,21(9):691-698.

8. Autar R.K.An Automated Diagnostic Expert System for Diesel Engines.Journal of Engineering for Gas Turbines and Power,1996,118:673-679.

9. Davia J,Krivine J,Tiarri J et al.DIVA Recognition of Typical Situations for Turbine Generator Diagnosis.Journal of Intelligent and Robotic Systems, 1988, 1:287-298. 